\title{
SELF-SIMILAR SOLUTIONS OF THE NON-STRICTLY HYPERBOLIC WHITHAM EQUATIONS*
}

\author{
VIRGIL U. PIERCE ${ }^{\dagger}$ AND FEI-RAN TIAN $\ddagger$
}

\begin{abstract}
We study the Whitham equations for the fifth order KdV equation. The equations are neither strictly hyperbolic nor genuinely nonlinear. We are interested in the solution of the Whitham equations when the initial values are given by a step function. We classify the step-like initial data into eight different types. We construct self-similar solutions for each type.
\end{abstract}

Key words. Zero dispersion limit, Whitham equations, non-stricitly hyperbolic equations

AMS subject classifications. 35L65, 35L67, 35Q05, 35Q15, 35Q53, 35Q58

\section{Introduction}

It is known that the solution of the $\mathrm{KdV}$ equation

$$
u_{t}+6 u u_{x}+\epsilon^{2} u_{x x x}=0
$$

has a weak limit as $\epsilon \rightarrow 0$ while the initial values

$$
u(x, 0 ; \epsilon)=u_{0}(x)
$$

are fixed.

The weak limit is described by hyperbolic equations. It satisfies the Burgers equation

$$
u_{t}+\left(3 u^{2}\right)_{x}=0
$$

until its solution develops shocks. Immediately after, the weak limit is governed by the Whitham equations $[4,5,12,13]$

$$
u_{i t}+\lambda_{i}\left(u_{1}, u_{2}, u_{3}\right) u_{i x}=0, \quad i=1,2,3,
$$

where the $\lambda_{i}$ 's are given by formulae (2.12). Equations (1.3) form a $3 \times 3$ system of hyperbolic equations. After the breaking of the solution of (1.3), the weak limit is described by a $5 \times 5$ system of hyperbolic equations similar to (1.3). Similarly, after the solution of the $5 \times 5$ system breaks down, the weak limit is characterized by a $7 \times 7$ system of hyperbolic equations. In other words, for general initial data $u_{0}(x)$, one must construct the weak limit by patching together solutions of $(1.2),(1.3), 5 \times 5$, $7 \times 7$, etc systems in the $x$ - $t$ plane.

The KdV equation (1.1) is just the first member of an infinite sequence of equations, the second of which is the so-called fifth order KdV equation

$$
u_{t}+30 u^{2} u_{x}+20 \epsilon^{2} u_{x} u_{x x}+10 \epsilon^{2} u u_{x x x}+\epsilon^{4} u_{x x x x x}=0 .
$$

*Received: July 26, 2006; accepted (in revised version): October 17, 2006. Communicated by Shi Jin.

$\dagger$ Department of Mathematics, Ohio State University, 231 W. 18th Avenue, Columbus, OH 43210, USA (vpierce@math.ohio-state.edu).

${ }^{\ddagger}$ Department of Mathematics, Ohio State University, 231 W. 18th Avenue, Columbus, OH 43210, USA (tian@math.ohio-state.edu). 
The solution of the fifth order KdV equation (1.4) also has a weak limit as $\epsilon \rightarrow 0$. As in the $\mathrm{KdV}$ case, this weak limit satisfies the Burgers type equation

$$
u_{t}+\left(10 u^{3}\right)_{x}=0
$$

until the solution of (1.5) forms a shock. After the breaking of the solution of (1.5), the limit is governed by equations similar to (1.3), namely,

$$
u_{i t}+\mu_{i}\left(u_{1}, u_{2}, u_{3}\right) u_{i x}=0, \quad i=1,2,3,
$$

where $\mu_{i}$ 's are given in (2.18). They will also be called the Whitham equations. As in the $\mathrm{KdV}$ case, after the solution of (1.6) breaks down, the weak limit is described by a $5 \times 5$ system of hyperbolic equations.

In this paper, we are interested in the solution of the Whitham equation (1.6) for the fifth order KdV (1.4) with a step-like initial function

$$
u_{0}(x)=\left\{\begin{array}{ll}
a, & x<0, \\
b, & x>0
\end{array} \quad a \neq b\right.
$$

For such an initial function with $a>0, b<a$ or $a<0, b>a$, the solution of the Burgers type equation (1.5) has already developed a shock at the initial time, $t=0$. Hence, immediately after $t=0$, the Whitham equations (1.6) kick in. Solutions of (1.6) occupy some domains of the space-time while solutions of (1.5) occupy other domains. These solutions are matched on the boundaries of the domains.

Equations (1.2) and equations (1.5) are prototypes in the theory of hyperbolic conservation laws [6]. Their solutions will generally develop shocks in finite times. The solutions can be extended beyond the singularities as the entropy solutions. The entropy solution of the Burgers equation (1.2) with initial function (1.7) is simple: it is either a rarefaction wave or a single shock wave. The Burgers type equation (1.5) is more complicated, as its flux function changes convexity at $u=0$. Its entropy solution with step-like initial data (1.7) can be a rarefaction wave, a single shock wave or a combination of both [6].

Solutions of equations (1.2) or equations (1.5), in the theory of the zero dispersion limit, are not extended as weak or entropy solutions after the formation of singularities. Instead, they are extended to match the Whitham solutions of (1.3) or (1.6). For initial data (1.7), the resulting solutions of the Whitham equations (1.6) will be seen to be more complex than those of (1.3) in the KdV case. Indeed, there are eight types of different solutions in the former case while there is only one type of solution in the latter case.

The KdV case with the step-like initial data (1.7) was first studied by Gurevich and Pitaevskii [2]. The Burgers solution of (1.2) develops a shock only for $a>b$. Moreover, because of the Galilean invariance of the $\mathrm{KdV}$ equation, the corresponding initial function is equivalent to the case $a=1, b=0$. In this case, Gurevich and Pitaevskii found that it was enough to use the Burgers solution of (1.2) and the Whitham solution of (1.3) to cover the whole $x$ - $t$ plane, without going to the $5 \times 5$ or $7 \times 7$ system. Namely, the space-time is divided into three parts

$$
\text { (1) } \frac{x}{t}<-6, \quad \text { (2) }-6<\frac{x}{t}<4, \quad \text { (3) } \frac{x}{t}>4 .
$$

The solution of (1.2) occupies the first and third parts,

$$
u(x, t) \equiv 1 \quad \text { when } \frac{x}{t}<-6, \quad u(x, t) \equiv 0 \quad \text { when } \frac{x}{t}>4 .
$$


The Whitham solution of (1.3) lives in the second part,

$$
u_{1}(x, t) \equiv 1, \quad \frac{x}{t}=\lambda_{2}\left(1, u_{2}, 0\right), \quad u_{3}(x, t) \equiv 0,
$$

when $-6<x / t<4$.

Whether the second equation of (1.9) can be inverted to give $u_{2}$ as a function of the self-similarity variable $x / t$ hinges on whether

$$
\frac{\partial \lambda_{2}}{\partial u_{2}}\left(1, u_{2}, 0\right) \neq 0
$$

Indeed, Levermore [7] has proved the genuine nonlinearity of the Whitham equations (1.3), i.e.,

$$
\frac{\partial \lambda_{i}}{\partial u_{i}}\left(u_{1}, u_{2}, u_{3}\right)>0, \quad i=1,2,3
$$

for $u_{1}>u_{2}>u_{3}$.

For the fifth order $\mathrm{KdV}$ (1.4), equations (1.6), in general, are not genuinely nonlinear, i.e., a property like (1.10) is not available. Hence, solutions like (1.8) and (1.9) need to be modified.

Our construction of solutions of the Whitham equation (1.6) makes use of the non-strict hyperbolicity of the equations. For $\mathrm{KdV}$, it is known that the Whitham equations (1.3) are strictly hyperbolic, namely:

$$
\lambda_{1}\left(u_{1}, u_{2}, u_{3}\right)>\lambda_{2}\left(u_{1}, u_{2}, u_{3}\right)>\lambda_{3}\left(u_{1}, u_{2}, u_{3}\right)
$$

for $u_{1}>u_{2}>u_{3}$. For the fifth order $\mathrm{KdV}$ (1.4), different eigenspeeds of (1.6), $\mu_{i}\left(u_{1}, u_{2}, u_{3}\right)$ 's, may coalesce in the region $u_{1}>u_{2}>u_{3}$.

For the fifth order $\mathrm{KdV}$ with step-like initial function (1.7) where $a=1$ and $b=0$, the space time is divided into four regions (see Figure 1.1)

$$
\text { (1) } \frac{x}{t}<-15, \quad(2)-15<\frac{x}{t}<\alpha, \quad \text { (3) } \alpha<\frac{x}{t}<16, \quad \text { (4) } \frac{x}{t}>16,
$$

where $\alpha$ is determined by (3.15). In the first and fourth regions, the solution of (1.5) governs the evolution:

$$
u(x, t) \equiv 1 \quad \text { where } x / t<-15 \text { and } u(x, t) \equiv 0 \quad \text { where } x / t>16 .
$$

The Whitham solution of (1.6) lives in the second and third regions; namely:

$$
u_{1}(x, t) \equiv 1, \quad \frac{x}{t}=\mu_{2}\left(1, u_{2}, u_{3}\right), \quad \frac{x}{t}=\mu_{3}\left(1, u_{2}, u_{3}\right),
$$

when $-15<x / t<\alpha$, and

$$
u_{1}(x, t) \equiv 1, \quad \frac{x}{t}=\mu_{2}\left(1, u_{2}, 0\right), \quad u_{3}(x, t) \equiv 0,
$$

when $\alpha<x / t<16$.

Equations (1.11) yield

$$
\mu_{2}\left(1, u_{2}, u_{3}\right)=\mu_{3}\left(1, u_{2}, u_{3}\right)
$$




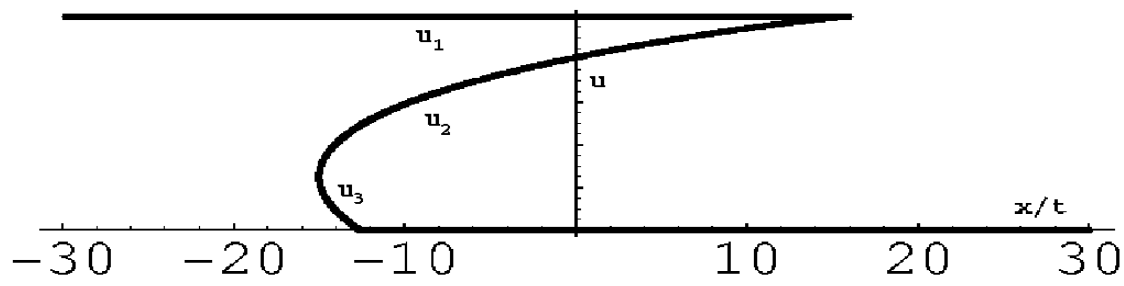

FIG. 1.1. Self-Similar solution of the Whitham equations for $a=1$ and $b=0$ of type II.

on a curve in the region $0<u_{3}<u_{2}<1$. This implies the non-strict hyperbolicity of the Whitham equations (1.6) for the fifth order KdV.

The organization of the paper is as follows. In Section 2, we will study the eigenspeeds, $\mu_{i}\left(u_{1}, u_{2}, u_{3}\right)$ 's, of the Whitham equations (1.6). In Section 3 , we will construct the self-similar solution of the Whitham equations for the initial function (1.7) with $a=1, b=0$. In Section 4, we will use the self-similar solution of Section 3 to construct the minimizer of a variational problem for the zero dispersion limit of the fifth order KdV. In Section 5, we will consider all the other possible step-like initial data (1.7). We find that there are eight different types of initial data. We construct self-similar solutions for each type.

\section{The Whitham equations}

In this section we define the eigenspeeds of the Whitham equations for both the $\mathrm{KdV}$ (1.1) and fifth order KdV (1.4). We first introduce the polynomials of $\xi$ for $n=0,1,2, \ldots[1,3,10]$ :

$$
P_{n}\left(\xi, u_{1}, u_{2}, u_{3}\right)=\xi^{n+1}+a_{n, 1} \xi^{n}+\cdots+a_{n, n+1},
$$

where the coefficients, $a_{n, 1}, a_{n, 2}, \ldots, a_{n, n+1}$ are uniquely determined by the two conditions

$$
\frac{P_{n}\left(\xi, u_{1}, u_{2}, u_{3}\right)}{\sqrt{\left(\xi-u_{1}\right)\left(\xi-u_{2}\right)\left(\xi-u_{3}\right)}}=\xi^{n-1 / 2}+\mathcal{O}\left(\xi^{-3 / 2}\right) \text { for large }|\xi|
$$

and

$$
\int_{u_{3}}^{u_{2}} \frac{P_{n}\left(\xi, u_{1}, u_{2}, u_{3}\right)}{\sqrt{\left(\xi-u_{1}\right)\left(\xi-u_{2}\right)\left(\xi-u_{3}\right)}} d \xi=0 .
$$

Here the sign of the square root is given by $\sqrt{\left(\xi-u_{1}\right)\left(\xi-u_{2}\right)\left(\xi-u_{3}\right)}>0$ for $\xi>u_{1}$ and the branch cuts are along $\left(-\infty, u_{3}\right)$ and $\left(u_{2}, u_{1}\right)$.

In particular,

$$
P_{0}\left(\xi, u_{1}, u_{2}, u_{3}\right)=\xi+a_{0,1}, \quad P_{1}\left(\xi, u_{1}, u_{2}, u_{3}\right)=\xi^{2}-\frac{1}{2}\left(u_{1}+u_{2}+u_{3}\right) \xi+a_{1,2}
$$


where

$$
\begin{aligned}
& a_{0,1}=\left(u_{1}-u_{3}\right) \frac{E(s)}{K(s)}-u_{1} \\
& a_{1,2}=\frac{1}{3}\left(u_{1} u_{2}+u_{1} u_{3}+u_{2} u_{3}\right)+\frac{1}{6}\left(u_{1}+u_{2}+u_{3}\right) a_{0,1}
\end{aligned}
$$

Here

$$
s=\frac{u_{2}-u_{3}}{u_{1}-u_{3}}
$$

and $K(s)$ and $E(s)$ are complete elliptic integrals of the first and second kind.

$K(s)$ and $E(s)$ have some well-known properties $[8,9]$. They have the expansions

$$
\begin{aligned}
& K(s)=\frac{\pi}{2}\left[1+\frac{s}{4}+\frac{9}{64} s^{2}+\cdots+\left(\frac{1 \cdot 3 \cdots(2 n-1)}{2 \cdot 4 \cdots 2 n}\right)^{2} s^{n}+\cdots\right] \\
& E(s)=\frac{\pi}{2}\left[1-\frac{s}{4}-\frac{3}{64} s^{2}-\cdots-\frac{1}{2 n-1}\left(\frac{1 \cdot 3 \cdots(2 n-1)}{2 \cdot 4 \cdots 2 n}\right)^{2} s^{n}-\cdots\right]
\end{aligned}
$$

for $|s|<1$. They also have the asymptotics

$$
\begin{aligned}
K(s) & \approx \frac{1}{2} \log \frac{16}{1-s}, \\
E(s) & \approx 1+\frac{1}{4}(1-s)\left[\log \frac{16}{1-s}-1\right],
\end{aligned}
$$

as $s$ is close to 1 . Furthermore,

$$
\begin{aligned}
\frac{d K(s)}{d s} & =\frac{E(s)-(1-s) K(s)}{2 s(1-s)}, \\
\frac{d E(s)}{d s} & =\frac{E(s)-K(s)}{2 s} .
\end{aligned}
$$

It immediately follows from (2.5) and (2.6) that

$$
\frac{1}{1-\frac{s}{2}}<\frac{K(s)}{E(s)}<\frac{1-\frac{s}{2}}{1-s} \quad \text { for } 0<s<1 .
$$

The eigenspeeds of the Whitham equations (1.3) are defined in terms of $P_{0}$ and $P_{1}$ of (2.4),

$$
\lambda_{i}\left(u_{1}, u_{2}, u_{3}\right)=12 \frac{P_{1}\left(u_{i}, u_{1}, u_{2}, u_{3}\right)}{P_{0}\left(u_{i}, u_{1}, u_{2}, u_{3}\right)}, \quad i=1,2,3,
$$

which give

$$
\begin{aligned}
& \lambda_{1}\left(u_{1}, u_{2}, u_{3}\right)=2\left(u_{1}+u_{2}+u_{3}\right)+4\left(u_{1}-u_{2}\right) \frac{K(s)}{E(s)}, \\
& \lambda_{2}\left(u_{1}, u_{2}, u_{3}\right)=2\left(u_{1}+u_{2}+u_{3}\right)+4\left(u_{2}-u_{1}\right) \frac{s K(s)}{E(s)-(1-s) K(s)}, \\
& \lambda_{3}\left(u_{1}, u_{2}, u_{3}\right)=2\left(u_{1}+u_{2}+u_{3}\right)+4\left(u_{2}-u_{3}\right) \frac{K(s)}{E(s)-K(s)} .
\end{aligned}
$$


Using (2.11), we obtain

$$
\begin{aligned}
& \lambda_{1}-2\left(u_{1}+u_{2}+u_{3}\right)>0, \\
& \lambda_{2}-2\left(u_{1}+u_{2}+u_{3}\right)<0, \\
& \lambda_{3}-2\left(u_{1}+u_{2}+u_{3}\right)<0,
\end{aligned}
$$

for $u_{1}>u_{2}>u_{3}$. In view of (2.5-2.8), we find that $\lambda_{1}, \lambda_{2}$ and $\lambda_{3}$ have behavior:

(1) At $u_{2}=u_{3}$ :

$$
\begin{aligned}
& \lambda_{1}\left(u_{1}, u_{2}, u_{3}\right)=6 u_{1}, \\
& \lambda_{2}\left(u_{1}, u_{2}, u_{3}\right)=\lambda_{3}\left(u_{1}, u_{2}, u_{3}\right)=12 u_{3}-6 u_{1} .
\end{aligned}
$$

(2) At $u_{1}=u_{2}$ :

$$
\begin{aligned}
& \lambda_{1}\left(u_{1}, u_{2}, u_{3}\right)=\lambda_{2}\left(u_{1}, u_{2}, u_{3}\right)=4 u_{1}+2 u_{3}, \\
& \lambda_{3}\left(u_{1}, u_{2}, u_{3}\right)=6 u_{3} .
\end{aligned}
$$

The eigenspeeds of the Whitham equations (1.6) are

$$
\mu_{i}\left(u_{1}, u_{2}, u_{3}\right)=80 \frac{P_{2}\left(u_{i}, u_{1}, u_{2}, u_{3}\right)}{P_{0}\left(u_{i}, u_{1}, u_{2}, u_{3}\right)}, \quad i=1,2,3 .
$$

They can be expressed in terms of $\lambda_{1}, \lambda_{2}$ and $\lambda_{3}$ of the KdV.

LEMma 2.1. [10] The eigenspeeds, $\mu_{i}$ 's, satisfy:

1 .

$$
\mu_{i}\left(u_{1}, u_{2}, u_{3}\right)=\frac{1}{2}\left[\lambda_{i}-2\left(u_{1}+u_{2}+u_{3}\right)\right] \frac{\partial q}{\partial u_{i}}\left(u_{1}, u_{2}, u_{3}\right)+q\left(u_{1}, u_{2}, u_{3}\right),
$$

where $q\left(u_{1}, u_{2}, u_{3}\right)$ is the solution of the boundary value problem of the EulerPoisson-Darboux equations:

$$
\begin{aligned}
2\left(u_{i}-u_{j}\right) \frac{\partial^{2} q}{\partial u_{i} \partial u_{j}} & =\frac{\partial q}{\partial u_{i}}-\frac{\partial q}{\partial u_{j}}, \quad i, j=1,2,3 ; i \neq j, \\
q(u, u, u) & =30 u^{2} .
\end{aligned}
$$

2.

$$
\frac{\partial \mu_{i}}{\partial u_{j}}=\frac{\frac{\partial \lambda_{i}}{\partial u_{j}}}{\lambda_{i}-\lambda_{j}}\left[\mu_{i}-\mu_{j}\right], \quad i \neq j .
$$

The solution of (2.20) is a symmetric quadratic function of $u_{1}, u_{2}$ and $u_{3}$

$$
q\left(u_{1}, u_{2}, u_{3}\right)=6\left(u_{1}^{2}+u_{2}^{2}+u_{3}^{2}\right)+4\left(u_{1} u_{2}+u_{1} u_{3}+u_{2} u_{3}\right) .
$$

For $\mathrm{KdV}, \lambda_{i}$ 's satisfy [8]

$$
\frac{\partial \lambda_{3}}{\partial u_{3}}<\frac{3}{2} \frac{\lambda_{2}-\lambda_{3}}{u_{2}-u_{3}}<\frac{\partial \lambda_{2}}{\partial u_{2}}
$$

for $u_{3}<u_{2}<u_{1}$. Similar results also hold for the fifth order KdV. 
LEMMA 2.2

$$
\begin{aligned}
& \frac{\partial \mu_{2}}{\partial u_{2}}>\frac{3}{2} \frac{\mu_{2}-\mu_{3}}{u_{2}-u_{3}} \quad \text { if } \frac{\partial q}{\partial u_{2}}>0 \\
& \frac{\partial \mu_{3}}{\partial u_{3}}<\frac{3}{2} \frac{\mu_{2}-\mu_{3}}{u_{2}-u_{3}} \quad \text { if } \frac{\partial q}{\partial u_{3}}>0
\end{aligned}
$$

for $u_{3}<u_{2}<u_{1}$.

Proof. We use (2.19) to calculate

$$
\begin{aligned}
\frac{\partial \mu_{3}}{\partial u_{3}} & =\frac{1}{2} \frac{\partial \lambda_{3}}{\partial u_{3}} \frac{\partial q}{\partial u_{3}}+\frac{1}{2}\left[\lambda_{3}-2\left(u_{1}+u_{2}+u_{3}\right)\right] \frac{\partial^{2} q}{\partial u_{3}^{2}} \\
& <\frac{3}{4} \frac{\lambda_{2}-\lambda_{3}}{u_{2}-u_{3}} \frac{\partial q}{\partial u_{3}}+\frac{1}{2}\left[\lambda_{3}-2\left(u_{1}+u_{2}+u_{3}\right)\right] \frac{\partial^{2} q}{\partial u_{3}^{2}}
\end{aligned}
$$

and

$$
\begin{aligned}
\mu_{2}-\mu_{3} & =\frac{1}{2}\left(\lambda_{2}-\lambda_{3}\right) \frac{\partial q}{\partial u_{3}}+\frac{1}{2}\left[\lambda_{3}-2\left(u_{1}+u_{2}+u_{3}\right)\right]\left(\frac{\partial q}{\partial u_{2}}-\frac{\partial q}{\partial u_{3}}\right) \\
& =\frac{1}{2}\left(\lambda_{2}-\lambda_{3}\right) \frac{\partial q}{\partial u_{3}}+\frac{1}{2}\left[\lambda_{3}-2\left(u_{1}+u_{2}+u_{3}\right)\right] 2\left(u_{2}-u_{3}\right) \frac{\partial^{2} q}{\partial u_{2} \partial u_{3}} \\
& =\frac{2}{3}\left(u_{2}-u_{3}\right)\left(\frac{3}{4} \frac{\lambda_{2}-\lambda_{3}}{u_{2}-u_{3}} \frac{\partial q}{\partial u_{3}}+\frac{3}{2}\left[\lambda_{3}-2\left(u_{1}+u_{2}+u_{3}\right)\right] \frac{\partial^{2} q}{\partial u_{2} \partial u_{3}}\right),
\end{aligned}
$$

where we have used equation (2.20)

$$
\frac{\partial q}{\partial u_{2}}-\frac{\partial q}{\partial u_{3}}=2\left(u_{2}-u_{3}\right) \frac{\partial^{2} q}{\partial u_{2} \partial u_{3}} .
$$

It follows from formula (2.22) for $q$ that

$$
3 \frac{\partial^{2} q}{\partial u_{2} \partial u_{3}}=\frac{\partial^{2} q}{\partial u_{3}^{2}}
$$

which, along with with (2.25) and (2.26), proves (2.23).

Inequality (2.24) can be proved in the same way.

The following calculations are useful in the subsequent sections.

Using formula (2.19) for $\mu_{2}$ and $\mu_{3}$ and formulae (2.12) for $\lambda_{2}$ and $\lambda_{3}$, we obtain

$$
\mu_{2}\left(u_{1}, u_{2}, u_{3}\right)-\mu_{3}\left(u_{1}, u_{2}, u_{3}\right)=\frac{2\left(u_{2}-u_{3}\right) K}{(K-E)[E-(1-s) K]} M\left(u_{1}, u_{2}, u_{3}\right),
$$

where

$$
M\left(u_{1}, u_{2}, u_{3}\right)=\left[\frac{\partial q}{\partial u_{3}}+(1-s) \frac{\partial q}{\partial u_{2}}\right] E-(1-s)\left(\frac{\partial q}{\partial u_{2}}+\frac{\partial q}{\partial u_{3}}\right) K .
$$

We then use (2.9), (2.10) and (2.22) to calculate

$$
\frac{\partial M\left(u_{1}, u_{2}, u_{3}\right)}{\partial u_{2}}=\frac{10\left(u_{1}-3 u_{2}-u_{3}\right)}{u_{1}-u_{3}}(E-K) .
$$


We next consider

$$
F\left(u_{1}, u_{2}, u_{3}\right):=\frac{\mu_{2}\left(u_{1}, u_{2}, u_{3}\right)-\mu_{3}\left(u_{1}, u_{2}, u_{3}\right)}{u_{2}-u_{3}} .
$$

Using formula (2.19) for $\mu_{2}$ and $\mu_{3}$ and formulae (2.12) for $\lambda_{2}$ and $\lambda_{3}$, we obtain

$$
\begin{aligned}
F & =-2 \frac{(1-s) K}{E-(1-s) K} \frac{\partial q}{\partial u_{2}}+2 \frac{K}{K-E} \frac{\partial q}{\partial u_{3}} \\
& =-4 \frac{s(1-s) K}{E-(1-s) K}\left(u_{1}-u_{3}\right) \frac{\partial^{2} q}{\partial u_{2} \partial u_{3}}+2\left[\frac{K}{K-E}-\frac{(1-s) K}{E-(1-s) K}\right] \frac{\partial q}{\partial u_{3}}
\end{aligned}
$$

where we have used equations (2.20) in the last equality. Finally, we use the expansions (2.5-2.6) for $K$ and $E$ to obtain

$$
\begin{aligned}
F\left(u_{1}, u_{2}, u_{3}\right) & =-4\left[\left(2-\frac{7}{4} s+\cdots\right)\left(u_{1}-u_{3}\right) \frac{\partial^{2} q}{\partial u_{2} \partial u_{3}}+\left(-\frac{3}{4}+O\left(s^{2}\right)\right) \frac{\partial q}{\partial u_{3}}\right] \\
& =-16\left[\left(2-\frac{7}{4} s+\cdots\right)\left(u_{1}-u_{3}\right)+\left(-\frac{3}{4}+O\left(s^{2}\right)\right)\left(u_{1}+u_{2}+3 u_{3}\right)\right],
\end{aligned}
$$

where we have used formula (2.22) for $q$ in the last equality.

\section{A Self-similar solution}

In this section, we construct the self-similar solution of the Whitham equations (1.6) for the initial function (1.7) with $a=1$ and $b=0$. We will study all the other step-like initial data in Section 5.

THEOREM 3.1. (see Figure 1.1) For the step-like initial data $u_{0}(x)$ of (1.7) with $a=1, b=0$, the solution of the Whitham equations (1.6) is given by

$$
u_{1}=1, \quad x=\mu_{2}\left(1, u_{2}, u_{3}\right) t, \quad x=\mu_{3}\left(1, u_{2}, u_{3}\right) t
$$

for $-15 t<x \leq \alpha t$ and by

$$
u_{1}=1, \quad x=\mu_{2}\left(1, u_{2}, 0\right) t, \quad u_{3}=0
$$

for $\alpha t \leq x<16 t$, where $\alpha=\mu_{2}\left(1, u^{*}, 0\right)$ and $u^{*}$ is the unique solution $u_{2}$ of $\mu_{2}\left(1, u_{2}, 0\right)=$ $\mu_{3}\left(1, u_{2}, 0\right)$ in the interval $0<u_{2}<1$. Outside the region $-15 t<x<16 t$, the solution of the Burgers type equation (1.5) is given by

$$
u \equiv 1 \quad x \leq-15 t
$$

and

$$
u \equiv 0 \quad x \geq 16 t .
$$

The boundaries $x=-15 t$ and $x=16 t$ are called the trailing and leading edges, respectively. They separate the solutions of the Whitham equations and Burgers type equations. The Whitham solution matches the Burgers type solution in the following fashion (see Figure 1.1):

$$
\begin{aligned}
& u_{1}=\text { the Burgers type solution defined outside the region, } \\
& u_{2}=u_{3},
\end{aligned}
$$


at the trailing edge;

$$
\begin{aligned}
& u_{1}=u_{2}, \\
& u_{3}=\text { the Burgers type solution defined outside the region, }
\end{aligned}
$$

at the leading edge.

The proof of Theorem 3.1 is based on a series of lemmas.

We first show that the solutions defined by formulae (3.1) and (3.2) indeed satisfy the Whitham equations $(1.6)[1,11]$.

LEMMA 3.2.

1. The functions $u_{1}, u_{2}$ and $u_{3}$ determined by equations (3.1) give a solution of the Whitham equations (1.6) as long as $u_{2}$ and $u_{3}$ can be solved from (3.1) as functions of $x$ and $t$.

2. The functions $u_{1}, u_{2}$ and $u_{3}$ determined by equations (3.2) give a solution of the Whitham equations (1.6) as long as $u_{2}$ can be solved from (3.2) as a function of $x$ and $t$.

Proof. (1) $u_{1}$ obviously satisfies the first equation of (1.6). To verify the second and third equations, we observe that

$$
\frac{\partial \mu_{2}}{\partial u_{3}}=\frac{\partial \mu_{3}}{\partial u_{2}}=0
$$

on the solution of (3.1). To see this, we use (2.21) to calculate

$$
\frac{\partial \mu_{2}}{\partial u_{3}}=\frac{\frac{\partial \lambda_{2}}{\partial u_{3}}}{\lambda_{2}-\lambda_{3}}\left(\mu_{2}-\mu_{3}\right)=0 .
$$

The second part of (3.9) can be shown in the same way.

We then calculate the partial derivatives of the second equation of (3.1) with respect to $x$ and $t$.

$$
1=\frac{\partial \mu_{2}}{\partial u_{2}} t u_{2 x}, \quad 0=\frac{\partial \mu_{2}}{\partial u_{2}} t u_{2 t}+\mu_{2},
$$

which give the second equation of (1.6).

The third equation of (1.6) can be verified in the same way.

(2) The second part of Lemma 3.2 can easily be proved.

We now determine the trailing edge. Eliminating $x$ and $t$ from the last two equations of (3.1) yields

$$
\mu_{2}\left(1, u_{2}, u_{3}\right)-\mu_{3}\left(1, u_{2}, u_{3}\right)=0 \text {. }
$$

Since it degenerates at $u_{2}=u_{3}$, we replace (3.10) by

$$
F\left(1, u_{2}, u_{3}\right):=\frac{\mu_{2}\left(1, u_{2}, u_{3}\right)-\mu_{3}\left(1, u_{2}, u_{3}\right)}{u_{2}-u_{3}}=0 .
$$

Here, the function $F$ is also defined in (2.29).

Therefore, at the trailing edge where $u_{2}=u_{3}$, i.e., $s=0$, equation (3.11), in view of the expansion (2.30), becomes

$$
2\left(1-u_{3}\right)-\frac{3}{4}\left(1+4 u_{3}\right)=0
$$


which gives $u_{2}=u_{3}=1 / 4$.

Lemma 3.3. Equation (3.11) has a unique solution satisfying $u_{2}=u_{3}$. The solution is $u_{2}=u_{3}=1 / 4$. The rest of equations (3.1) at the trailing edge are $u_{1}=1$ and $x / t=$ $\mu_{2}(1,1 / 4,1 / 4)=-15$.

Having located the trailing edge, we now solve equations (3.1) in the neighborhood of the trailing edge. We first consider equation (3.11). We use (2.30) to differentiate $F$ at the trailing edge $u_{1}=1, u_{2}=u_{3}=1 / 4$

$$
\frac{\partial F\left(1, \frac{1}{4}, \frac{1}{4}\right)}{\partial u_{2}}=\frac{\partial F\left(1, \frac{1}{4}, \frac{1}{4}\right)}{\partial u_{3}}=40,
$$

which show that equation (3.11) or equivalently (3.10) can be inverted to give $u_{3}$ as a decreasing function of $u_{2}$

$$
u_{3}=A\left(u_{2}\right)
$$

in a neighborhood of $u_{2}=u_{3}=1 / 4$.

We now extend the solution (3.12) of equation (3.10) in the region $1>u_{2}>1 / 4>$ $u_{3}>0$ as far as possible. We deduce from Lemma 2.2 that

$$
\frac{\partial \mu_{2}}{\partial u_{2}}>0, \quad \frac{\partial \mu_{3}}{\partial u_{3}}<0
$$

on the solution of (3.10). Because of (3.9) and (3.13), solution (3.12) of equation (3.10) can be extended as long as $1>u_{2}>1 / 4>u_{3}>0$.

There are two possibilities: (1) $u_{2}$ touches 1 before or simultaneously as $u_{3}$ reaches 0 and $(2) u_{3}$ touches 0 before $u_{2}$ reaches 1 .

It follows from (2.17) and (2.19) that

$$
\mu_{2}\left(1,1, u_{3}\right)>\mu_{3}\left(1,1, u_{3}\right) \quad \text { for } 0 \leq u_{3}<1 .
$$

This shows that (1) is impossible. Hence, $u_{3}$ will touch 0 before $u_{2}$ reaches 1 . When this happens, equation (3.10) becomes

$$
\mu_{2}\left(1, u_{2}, 0\right)-\mu_{3}\left(1, u_{2}, 0\right)=0 .
$$

Lemma 3.4. Equation (3.14) has a simple zero in the region $0<u_{2}<1$, counting multiplicities. Denoting the zero by $u^{*}$, then $\mu_{2}\left(1, u_{2}, 0\right)-\mu_{3}\left(1, u_{2}, 0\right)$ is positive for $u_{2}>u^{*}$ and negative for $u_{2}<u^{*}$.

Proof. We now use (2.27) and (2.28) to prove the lemma. In equation (2.27), $K-E$ and $E-(1-s) K$ are all positive for $0<s<1$ in view of (2.11). By (2.28),

$$
\frac{\partial M\left(1, u_{2}, 0\right)}{\partial u_{2}}=10\left(3 u_{2}-1\right)[K-E] \quad \text { for } 0<u_{2}<1 .
$$

Since $M\left(1, u_{2}, 0\right)$ vanishes at $u_{2}=0$ and is positive at $u_{2}=1$ in view of $(2.5-2.8)$, we conclude from the above derivative that $M\left(1, u_{2}, 0\right)$ has a simple zero in $0<u_{2}<1$. This zero is exactly $u^{*}$ and the rest of the theorem can be proved easily. 
Having solved equation (3.10) for $u_{3}$ as a decreasing function of $u_{2}$ for $1 / 4 \leq u_{2} \leq$ $u^{*}$, we turn to equations (3.1). Because of (3.13), the second equation of (3.1) gives $u_{2}$ as an increasing function of $x / t$, for $-15 \leq x / t \leq \alpha$, where

$$
\alpha=\mu_{2}\left(1, u^{*}, 0\right) .
$$

Consequently, $u_{3}$ is a decreasing function of $x / t$ in the same interval.

LEMMA 3.5. The last two equations of (3.1) can be inverted to give $u_{2}$ and $u_{3}$ as increasing and decreasing functions, respectively, of the self-similarity variable $x / t$ in the interval $-15 \leq x / t \leq \alpha$, where $\alpha=\mu_{2}\left(1, u^{*}, 0\right)$ and $u^{*}$ is given in Lemma 3.4.

We now turn to equations (3.2). We want to solve the second equation when $x / t>$ $\alpha$ or equivalently when $u_{2}>u^{*}$. According to Lemma $3.4, \mu_{2}\left(1, u_{2}, 0\right)-\mu_{3}\left(1, u_{2}, 0\right)>0$ for $u^{*}<u_{2}<1$, which, together with (2.23), shows that

$$
\frac{\partial \mu_{2}\left(1, u_{2}, 0\right)}{\partial u_{2}}>0
$$

Hence, the second equation of (3.2) can be solved for $u_{2}$ as an increasing function of $x / t$ as long as $u^{*}<u_{2}<1$. When $u_{2}$ reaches 1 , we have

$$
x / t=\mu_{2}(1,1,0)=16,
$$

where we have used (2.17) and (2.19) in the last equality. We have therefore proved the following result.

LEMMA 3.6. The second equation of (3.2) can be inverted to give $u_{2}$ as an increasing function of $x / t$ in the interval $\alpha \leq x / t \leq 16$.

We are ready to conclude the proof of Theorem 3.1.

The Burgers type solutions (3.3) and (3.4) are trivial.

According to Lemma 3.5, the last two equations of (3.1) determine $u_{2}$ and $u_{3}$ as functions of $x / t$ in the region $-15 \leq x / t \leq \alpha$. By the first part of Lemma 3.2, the resulting $u_{1}, u_{2}$ and $u_{3}$ satisfy the Whitham equations (1.6). Furthermore, the boundary conditions (3.5) and (3.6) are satisfied at the trailing edge $x=-15 t$.

Similarly, by Lemma 3.6, the second equation of (3.2) determines $u_{2}$ as a function of $x / t$ in the region $\alpha \leq x / t \leq 16$. It then follows from the second part of Lemma 3.2 that $u_{1}, u_{2}$ and $u_{3}$ of (3.2) satisfy the Whitham equations (1.6). They also satisfy the boundary conditions (3.7) and (3.8) at the leading edge $x=16 t$.

We have therefore completed the proof of Theorem 3.1.

A graph of the Whitham solution is given in Figure 1.1. It is obtained by plotting the exact solutions of (3.1) and (3.2).

\section{The Minimization problem}

The zero dispersion limit of the solution of the fifth order KdV equation (1.4) with step-like initial function (1.7), $a=1, b=0$, is also determined by a minimization problem with constraints $[4,5,12]$

$$
\underset{\left\{\psi \geq 0, \psi \in L^{1}\right\}}{\operatorname{Minimize}}\left\{-\frac{1}{2 \pi} \int_{0}^{1} \int_{0}^{1} \log \left|\frac{\eta-\mu}{\eta+\mu}\right| \psi(\eta) \psi(\mu) d \eta d \mu+\int_{0}^{1}\left[\eta x-16 \eta^{5} t\right] \psi(\eta) d \eta\right\} .
$$

In this section, we will use the self-similar solution of Section 3 to construct the minimizer. We first define a linear operator

$$
L \psi(\eta)=\frac{1}{2 \pi} \int_{0}^{1} \log \left(\frac{\eta-\mu}{\eta+\mu}\right)^{2} \psi(\mu) d \mu .
$$


The variational conditions are

$$
\begin{array}{ll}
L \psi=x \eta-16 t \eta^{5} & \text { where } \psi>0, \\
L \psi \leq x \eta-16 t \eta^{5} & \text { where } \psi=0 .
\end{array}
$$

The constraint for the minimization problem is

$$
\psi \geq 0
$$

The minimizer of (4.1) is given explicitly:

THEOREM 4.1. The minimizer of the variational problem (4.1) is as follows:

1. For $x \leq-15 t$,

$$
\psi(\eta)=\frac{-x \eta+80 t \eta\left(\eta^{4}-\frac{1}{2} \eta^{2}-\frac{1}{8}\right)}{\sqrt{1-\eta^{2}}}
$$

2. For $-15 t<x<\alpha t$,

$$
\psi(\eta)=\left\{\begin{array}{cc}
-\frac{-x \eta P_{0}\left(\eta^{2}, 1, u_{2}, u_{3}\right)+80 t \eta P_{2}\left(\eta^{2}, 1, u_{2}, u_{3}\right)}{\sqrt{\left(1-\eta^{2}\right)\left(u_{2}-\eta^{2}\right)\left(u_{3}-\eta^{2}\right)}}, & \eta<\sqrt{u_{3}}, \\
0, & \sqrt{u_{3}}<\eta<\sqrt{u_{2}}, \\
\frac{-x \eta P_{0}\left(\eta^{2}, 1, u_{2}, 0\right)+80 t \eta P_{2}\left(\eta^{2}, 1, u_{2}, 0\right)}{\sqrt{\left(1-\eta^{2}\right)\left(\eta^{2}-u_{2}\right)\left(\eta^{2}-u_{3}\right)}}, & \sqrt{u_{2}}<\eta<1,
\end{array}\right.
$$

where $P_{0}$ and $P_{2}$ are defined in (2.1) and $u_{2}$ and $u_{3}$ are determined by equations (3.1).

3. For $\alpha t<x<16 t$,

$$
\psi(\eta)=\left\{\begin{array}{cc}
0, & \eta<\sqrt{u_{2}}, \\
\frac{-x P_{0}\left(\eta^{2}, 1, u_{2}, 0\right)+80 t P_{2}\left(\eta^{2}, 1, u_{2}, 0\right)}{\sqrt{\left(1-\eta^{2}\right)\left(\eta^{2}-u_{2}\right)}}, & \sqrt{u_{2}}<\eta<1,
\end{array}\right.
$$

where $u_{2}$ is determined by (3.2).

4. For $x \geq 16 t$,

$$
\psi(\eta) \equiv 0
$$

Proof. We extend the function $\psi$ defined on $[0,1]$ to the entire real line by setting $\psi(\eta)=0$ for $\eta>1$ and taking $\psi$ to be odd. In this way, the operator $L$ is connected to the Hilbert transform $H$ on the real line [4]:

$$
L \psi(\eta)=\int_{0}^{\eta} H \psi(\mu) d \mu \quad \text { where } H \psi(\eta)=\frac{1}{\pi} P . V . \int_{-\infty}^{+\infty} \frac{\psi(\mu)}{\eta-\mu} d \mu .
$$

We verify case (4) first. Clearly, $\psi(\eta)=0$ satisfies the constraints (4.4). We now check the variational conditions (4.2-4.3). Since $\psi=0$,

$$
L \psi=0 \leq x \eta-16 t \eta^{5}
$$

where the inequality follows from $x \geq 16 t$ and $0 \leq \eta \leq 1$. Hence, variational conditions $(4.2-4.3)$ are satisfied. 
Next we consider case $(1)$. We write $\psi(\eta)$ as the real part of $g_{1}(\eta)$ for real $\eta$, where

$$
g_{1}=\sqrt{-1}\left(x-80 t \eta^{4}\right)+\frac{\sqrt{-1}\left[-x \eta+80 t \eta\left(\eta^{4}-\frac{1}{2} \eta^{2}-\frac{1}{8}\right)\right]}{\sqrt{\eta^{2}-1}} .
$$

The function $g_{1}$ is analytic in the upper half complex plane $\operatorname{Im}(\eta)>0$ and $g_{1}(\eta) \approx$ $O\left(1 / \eta^{2}\right)$ for large $|\eta|$. Hence, $H \psi(\eta)=\operatorname{Im}\left[g_{1}(\eta)\right]=x-80 t \eta^{4}$ on $0 \leq \eta \leq 1$, where $H$ is the Hilbert transform [4]. We then have for $0 \leq \eta \leq 1$

$$
L \psi(\eta)=\int_{0}^{\eta} H \eta(\mu) d \mu=x \eta-16 t \eta^{5},
$$

which shows that the variational conditions are satisfied. Since $15+80\left(\eta^{4}-\frac{1}{2} \eta^{2}-\frac{1}{8}\right)=$ $80\left(\eta^{2}-\frac{1}{4}\right)^{2} \geq 0$, it follows from $x \leq-15 t$ that $\psi \geq 0$. Hence, the constraint (4.4) is verified.

We now turn to case (2). By Lemma 3.5, the last two equations of (3.1) determine $u_{2}$ and $u_{3}$ as functions of the self-similarity variable $x / t$ in the interval $-15 \leq x / t \leq \alpha$.

We write $\psi=\operatorname{Re}\left(g_{2}\right)$ for real $\eta$, where

$$
g_{2}=\sqrt{-1}\left(x-80 t \eta^{4}\right)+\frac{\sqrt{-1}\left[-x \eta P_{0}\left(\eta^{2}, 1, u_{2}, u_{3}\right)+80 t \eta P_{2}\left(\eta^{2}, 1, u_{2}, u_{3}\right)\right]}{\sqrt{\left(\eta^{2}-1\right)\left(\eta^{2}-u_{2}\right)\left(\eta^{2}-u_{3}\right)}} .
$$

The function $g_{2}$ is analytic in $\operatorname{Im}(\eta)>0$ and $g_{2}(\eta) \approx O\left(1 / \eta^{2}\right)$ for large $|\eta|$ in view of the asymptotics (2.2) for $P_{0}$ and $P_{2}$. Hence, taking the imaginary part of $g_{2}$ yields

$$
H \psi(\eta)=\left\{\begin{array}{cc}
x-80 t \eta^{4}, & 0<\eta<\sqrt{u_{3}}, \\
x-80 t \eta^{4}-\frac{\left[-x P_{0}\left(\eta^{2}, 1, u_{2}, 0\right)+80 t P_{2}\left(\eta^{2}, 1, u_{2}, 0\right)\right] \eta}{\sqrt{\left(1-\eta^{2}\right)\left(u_{2}-\eta^{2}\right)\left(\eta^{2}-u_{3}\right)}}, & \sqrt{u_{3}}<\eta<\sqrt{u_{2}}, \\
x-80 t \eta^{4}, & \sqrt{u_{2}}<\eta<1 .
\end{array}\right.
$$

We then have

$$
L \psi(\eta)=\left\{\begin{array}{cc}
x \eta-16 t \eta^{5}, & 0<\eta<\sqrt{u_{3}}, \\
x \eta-16 t \eta^{5}-\int_{\sqrt{u_{3}} \frac{\left[-x P_{0}+80 t P_{2}\right] \mu}{\sqrt{\left(1-\mu^{2}\right)\left(u_{2}-\mu^{2}\right)\left(\mu^{2}-u_{3}\right)}} d \mu,} & \sqrt{u_{3}}<\eta<\sqrt{u_{2}}, \\
x \eta-16 t \eta^{5}, & \sqrt{u_{2}}<\eta<1 .
\end{array}\right.
$$

where we have used

$$
\int_{\sqrt{u_{3}}}^{\sqrt{u_{2}}} \frac{\left[-x P_{0}+80 t P_{2}\right] \mu}{\sqrt{\left(1-\mu^{2}\right)\left(u_{2}-\mu^{2}\right)\left(\mu^{2}-u_{3}\right)}} d \mu=0
$$

which is a consequence of (2.3) for $P_{0}$ and $P_{2}$.

We study the zeros of $-x P_{0}+80 t P_{2}$. It has two zeros at $\eta=\sqrt{u_{2}}$ and $\eta=\sqrt{u_{3}}$. This follows from (2.18) and (3.1). It also has a zero between $\sqrt{u_{2}}$ and $\sqrt{u_{3}}$ because of (4.5). Since it is a cubic polynomial of $\eta^{2},-x P_{0}+80 t P_{2}$ has no more than three zeros on the positive $\eta$ axis and furthermore these three positive zeros are simple.

Since the leading term in $-x P_{0}+80 t P_{2}$ is $80 t \eta^{6}$, the polynomial is positive for $\eta>\sqrt{u_{2}}$ and negative for $0 \leq \eta<\sqrt{u_{3}}$. This proves $\psi \geq 0$; so (4.4) is verified. Since $-x P_{0}+80 t P_{2}$ changes sign at each simple zero, it follows from (4.5) that

$$
\int_{\sqrt{u_{3}}}^{\eta} \frac{\left[-x P_{0}+80 t P_{2}\right] \mu}{\sqrt{\left(1-\mu^{2}\right)\left(u_{2}-\mu^{2}\right)\left(\mu^{2}-u_{3}\right)}} d \mu>0
$$


for $\sqrt{u_{3}}<\eta<\sqrt{u_{2}}$. This verifies the variational conditions (4.2) and (4.3).

We finally consider case (3). By Lemma 3.6, the second equation of (3.2) determines $u_{2}$ as an increasing function of $x / t$ in the interval $\alpha \leq x / t \leq 16$.

We write $\psi=\operatorname{Re}\left(g_{3}\right)$ for real $\eta$, where

$$
g_{3}=\sqrt{-1}\left(x-80 t \eta^{4}\right)+\frac{\sqrt{-1}\left[-x P_{0}\left(\eta^{2}, 1, u_{2}, 0\right)+80 t P_{2}\left(\eta^{2}, 1, u_{2}, 0\right)\right]}{\sqrt{\left(\eta^{2}-1\right)\left(\eta^{2}-u_{2}\right)}} .
$$

The function $g_{3}$ is analytic in $\operatorname{Im}(\eta)>0$ and $g_{3}(\eta) \approx O\left(1 / \eta^{2}\right)$ for large $|\eta|$ in view of the asymptotics $(2.2)$ for $P_{0}$ and $P_{2}$. Hence, taking the imaginary part of $g_{3}$ yields

$$
H \psi(\eta)=\left\{\begin{array}{cc}
x-80 t \eta^{4}-\frac{-x P_{0}\left(\eta^{2}, 1, u_{2}, 0\right)+80 t P_{2}\left(\eta^{2}, 1, u_{2}, 0\right)}{\sqrt{\left(1-\eta^{2}\right)\left(u_{2}-\eta^{2}\right)}}, & 0<\eta<\sqrt{u_{2}}, \\
x-80 t \eta^{4}, & \sqrt{u_{2}}<\eta<1 .
\end{array}\right.
$$

We then have

$$
L \psi(\eta)=\left\{\begin{array}{cc}
x \eta-16 t \eta^{5}-\int_{0}^{\eta} \frac{-x P_{0}+80 t P_{2}}{\sqrt{\left(1-\mu^{2}\right)\left(u_{2}-\mu^{2}\right)}} d \mu, & 0<\eta<\sqrt{u_{2}}, \\
x \eta-16 t \eta^{5}, & \sqrt{u_{2}}<\eta<1 .
\end{array}\right.
$$

where we have used

$$
\int_{0}^{\sqrt{u_{2}}} \frac{-x P_{0}\left(\mu^{2}, 1, u_{2}, 0\right)+80 t P_{2}\left(\mu^{2}, 1, u_{2}, 0\right)}{\sqrt{\left(1-\mu^{2}\right)\left(u_{2}-\mu^{2}\right)}} d \mu=0,
$$

which is a consequence of $(2.3)$ for $P_{0}$ and $P_{2}$.

The function $-x P_{0}\left(\eta^{2}, 1, u_{2}, 0\right)+80 t P_{2}\left(\eta^{2}, 1, u_{2}, 0\right)$ has two zeros on the positive $\eta$-axis. One is at $\eta=\sqrt{u_{2}}$, in view of (2.18) and (3.2). The other is between 0 and $\sqrt{u_{2}}$, in view of (4.6). At $\eta=0$, the function has a positive value. To see this,

$$
-x P_{0}\left(0,1, u_{2}, 0\right)+80 t P_{2}\left(0,1, u_{2}, 0\right)=P_{0}\left(0,1, u_{2}, 0\right)\left[-x+t \mu_{3}\left(1, u_{2}, 0\right)\right] .
$$

According to Lemma $3.4, \mu_{2}\left(1, u_{2}, 0\right)>\mu_{3}\left(1, u_{2}, 0\right)$ when $u_{2}>u^{*}$ or equivalently when $\alpha<x / t<16$. It follows from formula $(2.4)$ and inequality $(2.11)$ that $P_{0}\left(0,1, u_{2}, 0\right)<0$. Hence, the right hand side of (4.7) is bigger than

$$
P_{0}\left(0,1, u_{2}, 0\right)\left[-x+t \mu_{2}\left(1, u_{2}, 0\right)\right]=0
$$

where the equality comes from (3.2). Since it is a cubic polynomial in $\eta^{2}$ and since it is positive for large $\eta>0$, the function $-x P_{0}\left(\eta^{2}, 1, u_{2}, 0\right)+80 t P_{2}\left(\eta^{2}, 1, u_{2}, 0\right)$ can have at most two zeros on the positive $\eta$-axis. Hence, the above two zeros are all simple zeros.

It now becomes straight forward to check the variational conditions (4.2-4.3) and the constraint (4.4), just as we do in case (2).

\section{Other step like initial data}

In this section, we will classify all types of step-like initial data (1.7) for equation (1.4). When $a=0$, since $b \neq 0$, the solution of (1.5) will never develop a shock. We therefore study the cases $a>0$ and $a<0$. In the former case, it is easy to check that, when $b>a$, the solution of equation (1.5) will never develop a shock; accordingly, we will restrict to $b<a$. Similarly, in the latter case, we will confine ourselves to $b>a$.

We will only present our proofs briefly, since they are, more or less, similar to those in Section 3. 


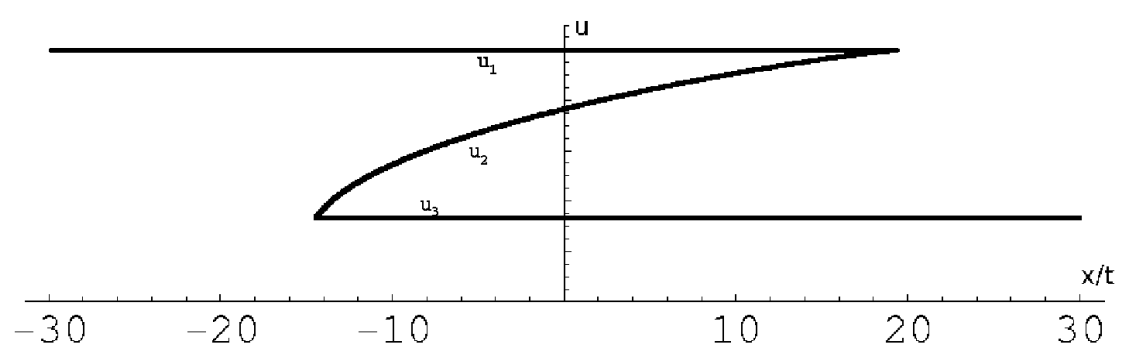

FIG. 5.1. Self-similar solution of the Whitham equations for $a=1$ and $b=1 / 3$ of type $I$.

5.1. Type I: $a>0, a / 4 \leq b<a$.

Theorem 5.1. (see Figure 5.1) For the step-like initial data (1.7) with $a>0, a / 4 \leq$ $b<a$, the solution of the Whitham equations (1.6) is given by

$$
u_{1}=a, \quad x=\mu_{2}\left(a, u_{2}, b\right) t, \quad u_{3}=b
$$

for $\mu_{2}(a, b, b)<x / t<\mu_{2}(a, a, b)$, where $\mu_{2}(a, b, b)=-10 a^{2}-40 a b+80 b^{2}$ and $\mu_{2}(a, a, b)=$ $16 a^{2}+8 a b+6 b^{2}$. Outside this interval, the solution of (1.5) is given by

$$
u \equiv a \quad x / t \leq \mu_{2}(a, b, b)
$$

and

$$
u \equiv b \quad x / t \geq \mu_{2}(a, a, b) .
$$

Proof. It suffices to show that $\mu_{2}\left(a, u_{2}, b\right)$ is an increasing function of $u_{2}$ for $b<u_{2}<a$. By (2.28), we have

$$
\frac{d M\left(a, u_{2}, b\right)}{d u_{2}}=\frac{10\left(3 u_{2}+b-a\right)}{a-b}[K-E]>0
$$

for $b<u_{2}<a$, where we have used $a / 4 \leq b<a$ in the inequality. Since $M\left(a, u_{2}, b\right)=0$ at $u_{2}=b$, this implies that $M\left(a, u_{2}, b\right)>0$ for $b<u_{2}<a$. It then follows from (2.27) that $\mu_{2}\left(a, u_{2}, b\right)-\mu_{3}\left(a, u_{2}, b\right)>0$. By Lemma 2.2 , we conclude that

$$
\frac{d \mu_{2}\left(a, u_{2}, b\right)}{d u_{2}}>0
$$

for $b<u_{2}<a$.

5.2. Type II: $a>0,-2 a / 3<b<a / 4$. Theorem 3.1 is a special case of the following theorem.

Theorem 5.2. (see Figure 1.1) For the step-like initial data (1.7) with $a>0,-2 a / 3<$ $b<a / 4$, the solution of the Whitham equations (1.6) is given by

$$
u_{1}=a, \quad x=\mu_{2}\left(a, u_{2}, u_{3}\right) t, \quad x=\mu_{3}\left(a, u_{2}, u_{3}\right) t
$$


for $-15 a^{2}<x / t \leq \mu_{2}\left(a, u^{* *}, b\right)$ and by

$$
u_{1}=a, \quad x=\mu_{2}\left(a, u_{2}, b\right) t, \quad u_{3}=b
$$

for $\mu_{2}\left(a, u^{* *}, b\right) \leq x / t<16 a^{2}+8 a b+6 b^{2}$, where $u^{* *}$ is the unique solution $u_{2}$ of $\mu_{2}\left(a, u_{2}, b\right)=\mu_{3}\left(a, u_{2}, b\right)$ in the interval $b<u_{2}<a$. Outside the region $-15 a^{2}<x / t<$ $16 a^{2}+8 a b+6 b^{2}$, the solution of the Burgers type equation (1.5) is given by

$$
u \equiv a \quad x / t \leq-15 a^{2}
$$

and

$$
u \equiv b \quad x / t \geq 16 a^{2}+8 a b+6 b^{2} .
$$

Proof. The trailing edge is determined by

$$
F\left(a, u_{2}, u_{3}\right)=0
$$

when $u_{2}=u_{3}$. Here $F$ is given by (2.29). In view of the expansion (2.30), the above equation when $u_{2}=u_{3}$, i.e., $s=0$, reduces to

$$
2\left(a-u_{3}\right)-\frac{3}{4}\left(a+4 u_{3}\right)=0
$$

which gives $u_{2}=u_{3}=a / 4$ at the trailing edge.

Having located the trailing edge, we solve equation (5.1) in the neighborhood of $u_{2}=u_{3}=a / 4$. We use the expansion (2.30) to calculate

$$
\frac{\partial F\left(a, \frac{a}{4}, \frac{a}{4}\right)}{\partial u_{2}}=\frac{\partial F\left(a, \frac{a}{4}, \frac{a}{4}\right)}{\partial u_{3}}=40,
$$

which implies that equation (5.1) can be solved for $u_{3}$ as a decreasing function of $u_{2}$ near $u_{2}=u_{3}=a / 4$.

The solution of

$$
\mu_{2}\left(a, u_{2}, u_{3}\right)-\mu_{3}\left(a, u_{2}, u_{3}\right)=0
$$

can be extended as long as $a>u_{2}>a / 4>u_{3}>b$. To see this, we need to show that

$$
\frac{\partial \mu_{2}\left(a, u_{2}, u_{3}\right)}{\partial u_{3}}=0, \quad \frac{\partial \mu_{3}\left(a, u_{2}, u_{3}\right)}{\partial u_{2}}=0, \quad \frac{\partial \mu_{2}\left(a, u_{2}, u_{3}\right)}{\partial u_{2}}>0, \quad \frac{\partial \mu_{3}\left(a, u_{2}, u_{3}\right)}{\partial u_{3}}<0
$$

on the solution of (5.2). The proof of the equalities is the same as that of (3.9) in Section 3. To prove the inequalities, in view of Lemma 2.2, it is enough to show that

$$
\frac{\partial q\left(a, u_{2}, u_{3}\right)}{\partial u_{2}}>0, \quad \frac{\partial q\left(a, u_{2}, u_{3}\right)}{\partial u_{3}}>0 .
$$

We use formulae (2.19) to rewrite equation (5.2) as

$$
\frac{1}{2}\left[\lambda_{2}-2\left(a+u_{2}+u_{3}\right)\right] \frac{\partial q\left(a, u_{2}, u_{3}\right)}{\partial u_{2}}=\frac{1}{2}\left[\lambda_{3}-2\left(a+u_{2}+u_{3}\right)\right] \frac{\partial q\left(a, u_{2}, u_{3}\right)}{\partial u_{3}},
$$


which, together with inequalities (2.14) and (2.15), proves that $\frac{\partial q}{\partial u_{2}}$ and $\frac{\partial q}{\partial u_{3}}$ have the same sign on the solution of (5.2). On the other hand, we calculate from (2.22)

$$
\frac{\partial q\left(a, u_{2}, u_{3}\right)}{\partial u_{2}}=4\left(a+3 u_{2}+u_{3}\right)>0
$$

for $a>u_{2}>a / 4>u_{3}>b>-2 a / 3$.

We now extend the solution of (5.2) as far as possible in the region $a>u_{2}>a / 4>$ $u_{3}>b$. There are two possibilities: (1) $u_{2}$ touches $a$ before or simultaneously as $u_{3}$ reaches $b$ and (2) $u_{3}$ touches $b$ before $u_{2}$ reaches $a$.

Possibility (1) is impossible. To see this, we use (2.17) and (2.19) to calculate

$$
\mu_{2}\left(a, a, u_{3}\right)-\mu_{3}\left(a, a, u_{3}\right)=2\left(a-u_{3}\right) \frac{\partial q\left(a, a, u_{3}\right)}{\partial u_{3}}=8\left(a-u_{3}\right)\left(2 a+3 u_{3}\right),
$$

which, in view of $b>-2 a / 3$, is positive for $b \leq u_{3}<a$.

Therefore, $u_{3}$ will touch $b$ before $u_{2}$ reaches $a$. When this happens, we have $\mu_{2}\left(a, u_{2}, b\right)-\mu_{3}\left(a, u_{2}, b\right)=0$. In the same way as we prove Lemma 3.4, we can show that this equation has a unique solution $u_{2}$ in the interval $b<u_{2}<a$.

The rest of the proof is similar to that of Theorem 3.1.

5.3. Type III: $a>0, b=-2 a / 3$.

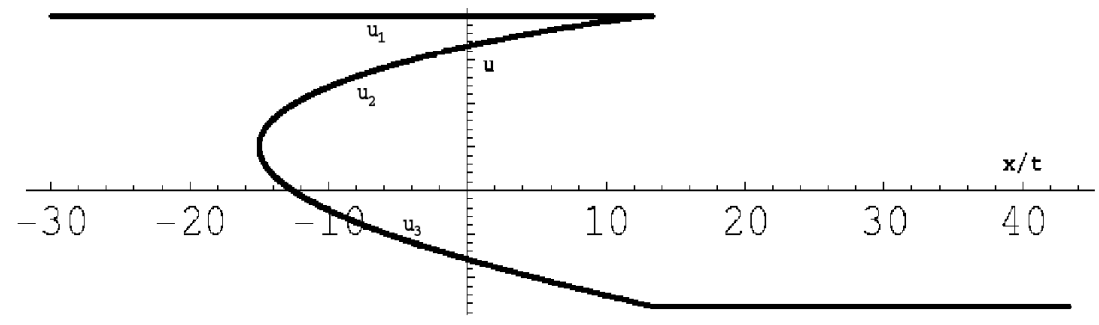

FIG. 5.2. Self-similar solution of the Whitham equations for $a=1$ and $b=-2 / 3$ of type III.

THEOREM 5.3. (see Figure 5.2) For the step-like initial data (1.7) with $a>0, b=$ $-2 a / 3$, the solution of the Whitham equations (1.6) is given by

$$
u_{1}=a, \quad x=\mu_{2}\left(a, u_{2}, u_{3}\right) t, \quad x=\mu_{3}\left(a, u_{2}, u_{3}\right) t
$$

for $-15 a^{2}<x / t<40 a^{2} / 3$. Outside the region, the solution of the Burgers type equation (1.5) is given by

$$
u \equiv a \quad x / t \leq-15 a^{2}
$$

and

$$
u \equiv b \quad x / t \geq 40 a^{2} / 3 .
$$


Proof. It suffices to show that $u_{2}$ and $u_{3}$ of $\mu_{2}\left(a, u_{2}, u_{3}\right)-\mu_{3}\left(a, u_{2}, u_{3}\right)=0$ reaches $a$ and $b=-2 a / 3$, respectively, simultaneously. To see this, we deduce from equation (5.3) that

$$
\mu_{2}(a, a,-2 a / 3)-\mu_{3}(a, a,-2 a / 3)=8(a-2 a / 3)[2 a+3(-2 a / 3)]=0 .
$$

5.4. Type IV: $a>0, b<-2 a / 3$.

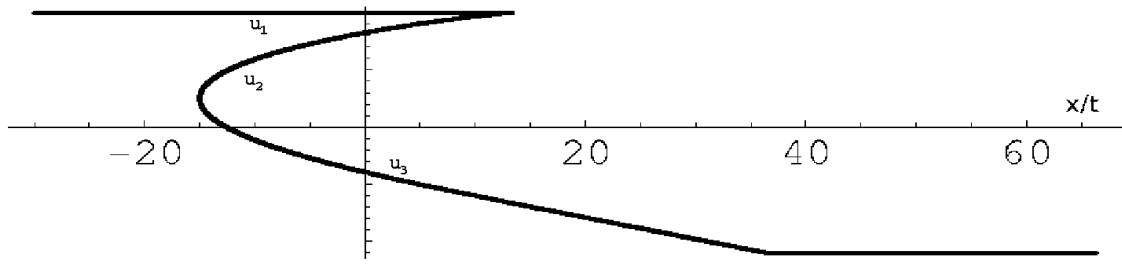

FIG. 5.3. Self-similar solution of the Whitham equations for $a=1$ and $b=-1.1$ of type $I V$.

Theorem 5.4. (see Figure 5.3) For the step-like initial data (1.7) with $a>0, b<$ $-2 a / 3$, the solution of the Whitham equations (1.6) is given by

$$
u_{1}=a, \quad x=\mu_{2}\left(a, u_{2}, u_{3}\right) t, \quad x=\mu_{3}\left(a, u_{2}, u_{3}\right) t
$$

for $-15 a^{2}<x / t<40 a^{2} / 3$. Outside the region, the solution of the Burgers type equation (1.5) is given by

$$
u \equiv a \quad x / t \leq-15 a^{2}
$$

and

$$
u=\left\{\begin{array}{cc}
-\sqrt{\frac{x}{30 t}} & 40 a^{2} / 3 \leq x / t \leq 30 b^{2} \\
b & x / t \geq 30 b^{2}
\end{array} .\right.
$$

Proof. By the calculation (5.4), when $u_{2}$ of $\mu_{2}\left(a, u_{2}, u_{3}\right)-\mu_{3}\left(a, u_{2}, u_{3}\right)=0$ touches $a$, the corresponding $u_{3}$ reaches $-2 a / 3$, which is above $b$. Hence, equations

$$
x=\mu_{2}\left(a, u_{2}, u_{3}\right) t, \quad x=\mu_{3}\left(a, u_{2}, u_{3}\right) t
$$

can be inverted to give $u_{2}$ and $u_{3}$ as functions of $x / t$ in the region $\mu_{2}(a, a / 4, a / 4)<$ $x / t \leq \mu_{2}(a, a,-2 a / 3)$. To the right of this region, the Burgers type equation (1.5) has a rarefaction wave solution.

5.5. Type V: $a<0, b \leq-a / 4$.

THEOREM 5.5. (see Figure 5.4) For the step-like initial data (1.7) with $a<0, a<b \leq$ $-a / 4$, the solution of the Whitham equations (1.6) is given by

$$
u_{1}=b, \quad x=\mu_{2}\left(b, u_{2}, a\right) t, \quad u_{3}=a
$$




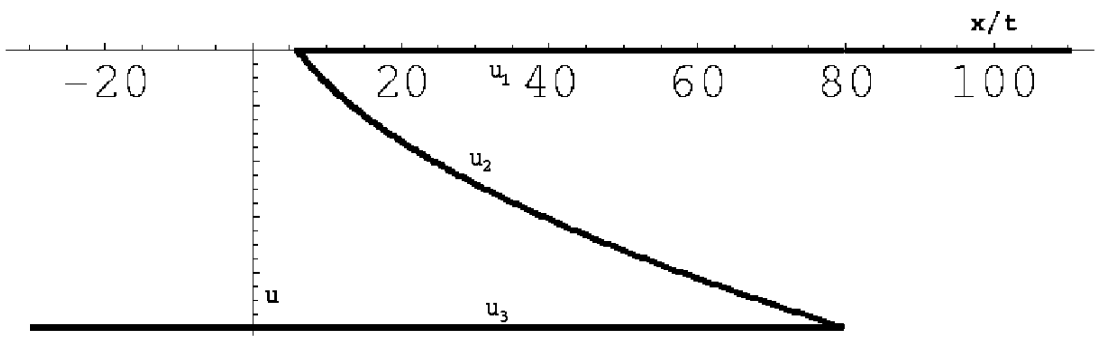

FIG. 5.4. Self-Similar solution of the Whitham equations for $a=-1$ and $b=0$ of type $V$.

for $\mu_{2}(b, b, a)<x / t<\mu_{2}(b, a, a)$, where $\mu_{2}(b, b, a)=6 a^{2}+8 a b+16 b^{2}$ and $\mu_{2}(b, a, a)=$ $80 a^{2}-40 a b-10 b^{2}$. Outside this interval, the solution of (1.5) is given by

$$
u \equiv a \quad x / t \leq \mu_{2}(b, b, a)
$$

and

$$
u \equiv b \quad x / t \geq \mu_{2}(b, a, a)
$$

Proof. It suffices to show that $\mu_{2}\left(a, u_{2}, b\right)$ is a decreasing function of $u_{2}$ for $a<$ $u_{2}<b$. By (2.19), we have

$$
\frac{\partial \mu_{2}\left(b, u_{2}, a\right)}{\partial u_{2}}=\frac{1}{2} \frac{\partial \lambda_{2}}{\partial u_{2}} \frac{\partial q}{\partial u_{2}}+\frac{1}{2}\left[\lambda_{2}-2\left(b+u_{2}+a\right)\right] \frac{\partial^{2} q}{\partial u_{2}^{2}} .
$$

The second term is negative because of $(2.14)$ and $\frac{\partial^{2} q}{\partial u_{2}^{2}}=12>0$. The first term is also negative. Its first factor is positive in view of (1.10). The second factor

$$
\frac{\partial q}{\partial u_{2}}=4\left(b+3 u_{2}+a\right)<0
$$

for $a<u_{2}<b$ because of $b \leq-a / 4$.

5.6. Type VI: $a<0,-a / 4<b<-2 a$.

Theorem 5.6. (see Figure 5.5) For the step-like initial data (1.7) with $a<0,-a / 4<$ $b<-2 a$, the solution of the Whitham equations (1.6) is given by

$$
x=\mu_{1}\left(u_{1}, u_{2}, a\right) t, \quad x=\mu_{2}\left(u_{1}, u_{2}, a\right) t, \quad u_{3}=a
$$

for $5 a^{2}<x / t \leq \mu_{2}\left(b, u^{* * *}, a\right)$ and by

$$
u_{1}=b, \quad x=\mu_{2}\left(b, u_{2}, a\right) t, \quad u_{3}=a
$$

for $\mu_{2}\left(b, u^{* * *}, a\right) \leq x / t<80 a^{2}-40 a b-10 b^{2}$, where $u^{* * *}$ is the unique solution $u_{2}$ of $\mu_{1}\left(b, u_{2}, a\right)=\mu_{2}\left(b, u_{2}, a\right)$ in the interval $a<u_{2}<b$. Outside the region $5 a^{2}<x / t<$ $80 a^{2}-40 a b-10 b^{2}$, the solution of the Burgers type equation (1.5) is given by

$$
u \equiv a \quad x / t \leq 5 a^{2}
$$




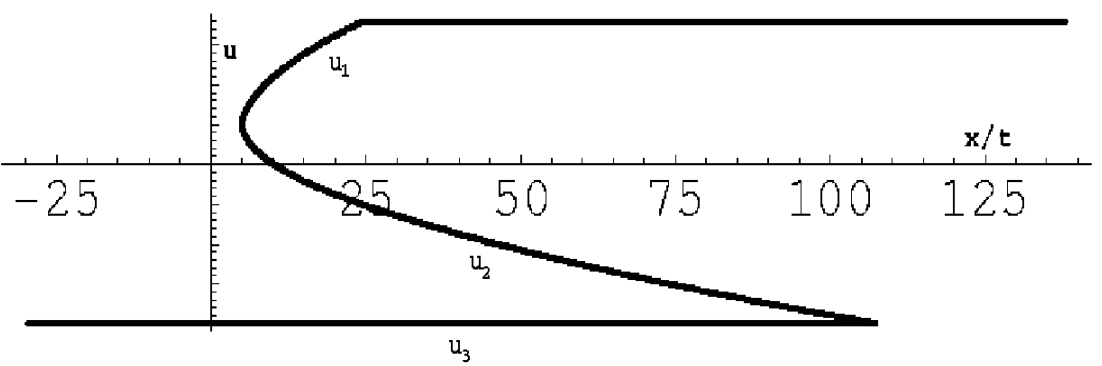

FIG. 5.5. Self-Similar solution of the Whitham equations for $a=-1$ and $b=1.2$ of type VI.

and

$$
u \equiv b \quad x / t \geq 80 a^{2}-40 a b-10 b^{2} .
$$

Proof. We first locate the "leading" edge, i.e., the solution of equation (5.5) at $u_{1}=u_{2}$. Eliminating $x / t$ from the first two equations of (5.5) yields

$$
\mu_{1}\left(u_{1}, u_{2}, a\right)-\mu_{2}\left(u_{1}, u_{2}, a\right)=0 .
$$

Since it degenerates at $u_{1}=u_{2}$, we replace (5.7) by

$$
G\left(u_{1}, u_{2}, a\right):=\frac{\mu_{1}\left(u_{1}, u_{2}, a\right)-\mu_{2}\left(u_{1}, u_{2}, a\right)}{\left(u_{1}-u_{2}\right) K(s)}=0 .
$$

Using formulae (2.19) for $\mu_{1}$ and $\mu_{2}$ and formulae (2.12) for $\lambda_{1}$ and $\lambda_{2}$, we write

$$
G\left(u_{1}, u_{2}, a\right)=\frac{2}{E[E-(1-s) K]}\left\{\left(\frac{\partial q}{\partial u_{1}}+s \frac{\partial q}{\partial u_{2}}\right) E-(1-s) \frac{\partial q}{\partial u_{1}} K\right\} .
$$

In view of (2.7) and (2.8), equation (5.8) reduces to

$$
\frac{\partial q\left(u_{1}, u_{2}, a\right)}{\partial u_{1}}+\frac{\partial q\left(u_{1}, u_{2}, a\right)}{\partial u_{2}}=0
$$

at the "leading" edge $u_{1}=u_{2}$. This gives

$$
u_{1}=u_{2}=-\frac{a}{4}
$$

Having located the "leading" edge, we solve equation (5.8) near $u_{1}=u_{2}=-a / 4$. We calculate

$$
\frac{\partial G(-a / 4,-a / 4, a)}{\partial u_{1}}=\frac{\partial G(-a / 4,-a / 4, a)}{\partial u_{2}}=32 .
$$

These show that equation (5.8) gives $u_{1}$ as a decreasing function of $u_{2}$

$$
u_{1}=B\left(u_{2}\right)
$$


in a neighborhood of $u_{1}=u_{2}=-a / 4$.

We now extend the solution (5.9) of equation (5.7) as far as possible in the region $a<u_{2}<-a / 4<u_{1}<b$. We use formula (2.19) to calculate

$$
\begin{aligned}
& \frac{\partial \mu_{1}}{\partial u_{1}}=\frac{1}{2} \frac{\partial \lambda_{1}}{\partial u_{1}} \frac{\partial q}{\partial u_{1}}+\frac{1}{2}\left[\lambda_{1}-2\left(u_{1}+u_{2}+a\right)\right] \frac{\partial^{2} q}{\partial u_{1}^{2}}, \\
& \frac{\partial \mu_{2}}{\partial u_{2}}=\frac{1}{2} \frac{\partial \lambda_{2}}{\partial u_{2}} \frac{\partial q}{\partial u_{2}}+\frac{1}{2}\left[\lambda_{2}-2\left(u_{1}+u_{2}+a\right)\right] \frac{\partial^{2} q}{\partial u_{2}^{2}} .
\end{aligned}
$$

In view of (1.10), (2.13) and (2.14), we have

$$
\begin{aligned}
& \frac{\partial \mu_{1}}{\partial u_{1}}>0 \text { if } \frac{\partial q}{\partial u_{1}}>0, \\
& \frac{\partial \mu_{2}}{\partial u_{2}}<0 \text { if } \frac{\partial q}{\partial u_{2}}<0 .
\end{aligned}
$$

We claim that

$$
\frac{\partial q}{\partial u_{1}}>0, \quad \frac{\partial q}{\partial u_{2}}<0
$$

on the solution of (5.7) in the region $a<u_{2}<-a / 4<u_{1}<b$. To see this, we use formula (2.19) to rewrite equation (5.7) as

$$
\frac{1}{2}\left[\lambda_{1}-2\left(u_{1}+u_{2}+a\right)\right] \frac{\partial q}{\partial u_{1}}=\frac{1}{2}\left[\lambda_{2}-2\left(u_{1}+u_{2}+a\right)\right] \frac{\partial q}{\partial u_{2}} .
$$

This, together with

$$
\frac{\partial q}{\partial u_{1}}-\frac{\partial q}{\partial u_{2}}=2\left(u_{1}-u_{2}\right) \frac{\partial^{2} q}{\partial u_{1} \partial u_{2}}=8\left(u_{1}-u_{2}\right)>0
$$

for $u_{1}>u_{2}$, and inequalities (2.13) and (2.14), proves (5.10).

Hence, the solution (5.9) can be extended as long as $a<u_{2}<-a / 4<u_{1}<b$.

There are two possibilities: (1) $u_{1}$ touches $b$ before $u_{2}$ reaches $a$ and (2) $u_{2}$ touches $a$ before or simultaneously as $u_{1}$ reaches $a$.

Possibility (2) is impossible. To see this, we use (2.16), (2.19) and (2.22) to calculate

$$
\mu_{1}\left(u_{1}, a, a\right)-\mu_{2}\left(u_{1}, a, a\right)=40\left(u_{1}-a\right)\left(u_{1}+2 a\right),
$$

which is negative for $-a / 4<u_{1} \leq b<-2 a$.

Therefore, $u_{1}$ will touch $b$ before $u_{2}$ reaches $a$. When this happens, we have

$$
\mu_{1}\left(b, u_{2}, a\right)-\mu_{2}\left(b, u_{2}, a\right)=0 .
$$

LEMMA 5.7. Equation (5.12) has a simple zero, counting multiplicities, in the interval $a<u_{2}<b$. Denoting this zero by $u^{* * *}$, then $\mu_{1}\left(b, u_{2}, a\right)-\mu_{2}\left(b, u_{2}, a\right)$ is positive for $u_{2}>u^{* * *}$ and negative for $u_{2}<u^{* * *}$.

Proof. We write

$$
\mu_{1}\left(b, u_{2}, a\right)-\mu_{2}\left(b, u_{2}, a\right)=\frac{2\left(b-u_{2}\right) K}{E[E-(1-s) K]}\left\{\left(\frac{\partial q}{\partial u_{1}}+s \frac{\partial q}{\partial u_{2}}\right) E-(1-s) \frac{\partial q}{\partial u_{1}} K\right\} .
$$


Denote the parenthesis of $(5.13)$ by $N\left(b, u_{2}, a\right)$. Since $E-(1-s) K>0$ for $a<u_{2}<b$, the left hand side has a zero iff $N\left(b, u_{2}, a\right)$ on the right has one.

We now calculate

$$
\frac{\partial N\left(b, u_{2}, a\right)}{\partial u_{2}}=\frac{30 E(s)}{b-a}\left[u_{2}-\frac{a-b}{3}\right]
$$

Since $N\left(b, u_{2}, a\right)$ is zero at $u_{2}=a$ and positive at $u_{2}=b$, we conclude from the above derivative that $N\left(b, u_{2}, a\right)$ has a simple zero in $a<u_{2}<b$.

We now continue to prove Theorem 5.6. Having solved equation (5.7) for $u_{1}$ as a decreasing function of $u_{2}$ for $u^{* * *}<u_{2}<-a / 4$, we can then use the last two equations of (5.5) to determine $u_{1}$ and $u_{2}$ as functions of $x / t$ in the interval $\mu_{2}(-a / 4,-a / 4, a)<$ $x / t<\mu_{2}\left(b, u^{* * *}, a\right)$.

We finally turn to equations (5.6). We want to solve the second equation of (5.6), $x / t=\mu_{2}\left(b, u_{2}, a\right)$, for $u_{2}<u^{* * *}$. It is enough to show that $\mu_{2}\left(b, u_{2}, a\right)$ is a decreasing function of $u_{2}$ for $u_{2}<u^{* * *}$.

According to Lemma 5.7, $\mu_{1}\left(b, u_{2}, a\right)-\mu_{2}\left(b, u_{2}, a\right)<0$ for $u_{2}<u^{* * *}$. Using formula (2.19) for $\mu_{1}$ and $\mu_{2}$, we have

$$
\frac{1}{2}\left[\lambda_{1}-2\left(b+u_{2}+a\right)\right] \frac{\partial q}{\partial u_{1}}<\frac{1}{2}\left[\lambda_{2}-2\left(b+u_{2}+a\right)\right] \frac{\partial q}{\partial u_{2}} .
$$

This, together with

$$
\frac{\partial q}{\partial u_{1}}-\frac{\partial q}{\partial u_{2}}=2\left(b-u_{2}\right) \frac{\partial^{2} q}{\partial u_{1} \partial u_{2}}=8\left(b-u_{2}\right)>0
$$

for $u_{1}>u_{2}$, and inequalities (2.13) and (2.14), proves

$$
\frac{\partial q\left(b, u_{2}, a\right)}{\partial u_{2}}<0
$$

for $u_{2}<u^{* * *}$. Hence,

$$
\frac{\partial \mu_{2}}{\partial u_{2}}=\frac{1}{2} \frac{\partial \lambda_{2}}{\partial u_{2}} \frac{\partial q}{\partial u_{2}}+\frac{1}{2}\left[\lambda_{2}-2\left(b+u_{2}+a\right)\right] \frac{\partial^{2} q}{\partial u_{2}^{2}}<0 .
$$

5.7. Type VII: $a<0, b=-2 a$.

THEOREM 5.8. (see Figure 5.6) For the step-like initial data (1.7) with $a<0, b=-2 a$, the solution of the Whitham equations (1.6) is given by

$$
x=\mu_{1}\left(u_{1}, u_{2}, a\right) t, \quad x=\mu_{2}\left(u_{1}, u_{2}, a\right) t, \quad u_{3}=a
$$

for $5 a^{2}<x / t<120 a^{2}$. Outside the region, the solution of the Burgers type equation (1.5) is given by

$$
u \equiv a \quad x / t \leq 5 a^{2}
$$

and

$$
u \equiv b \quad x / t \geq 120 a^{2}
$$




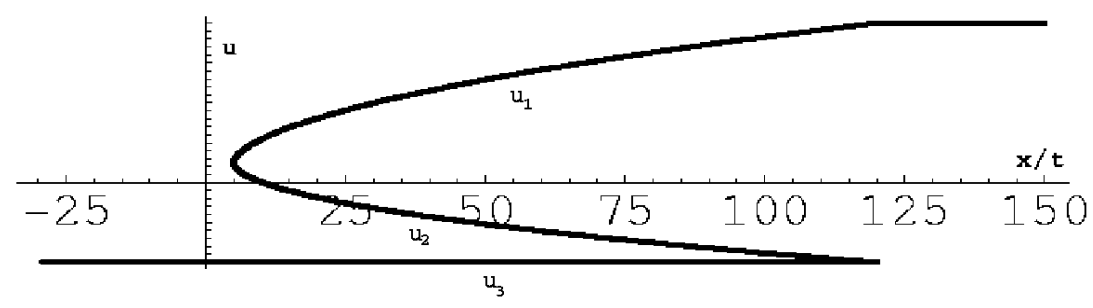

FIG. 5.6. Self-Similar solution of the Whitham equations for $a=-1$ and $b=2$ of type VII.

Proof. It suffices to show that $u_{1}$ and $u_{2}$ of $\mu_{1}\left(u_{1}, u_{2}, a\right)-\mu_{2}\left(u_{1}, u_{2}, a\right)=0$ reaches $b=-2 a$ and $a$, respectively, simultaneously. To see this, we deduce from equation (5.11) that

$$
\mu_{1}\left(u_{1}, a, a\right)-\mu_{3}\left(u_{1}, a, a\right)=8\left(u_{1}-a\right)\left(u_{1}+2 a\right)
$$

is negative for $u_{1}<b$ and vanish when $u_{1}=b=-2 a$.

5.8. Type VIII: $a<0, b>-2 a$.

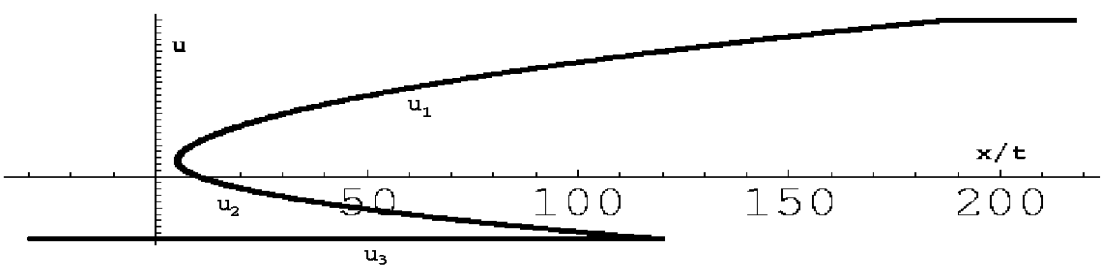

FIG. 5.7. Self-Similar solution of the Whitham equations for $a=-1$ and $b=2.5$ of type VIII.

THEOREM 5.9. (see Figure 5.7) For the step-like initial data (1.7) with $a<0, b>-2 a$, the solution of the Whitham equations (1.6) is given by

$$
x=\mu_{1}\left(u_{1}, u_{2}, a\right) t, \quad x=\mu_{2}\left(u_{1}, u_{2}, a\right) t, \quad u_{3}=a
$$

for $5 a^{2}<x / t<120 a^{2}$. Outside the region, the solution of the Burgers type equation (1.5) is given by

$$
u \equiv a, \quad x / t \leq 5 a^{2},
$$

and

$$
u=\left\{\begin{array}{cc}
\sqrt{\frac{x}{30 t}}, & 120 a^{2} \leq x / t \leq 30 b^{2} \\
b, & x / t \geq 30 b^{2} .
\end{array}\right.
$$


Proof. By the calculation (5.14), when $u_{2}$ of $\mu_{2}\left(u_{1}, u_{2}, a\right)-\mu_{3}\left(u_{1}, u_{2}, a\right)=0$ touches $a$, the corresponding $u_{3}$ reaches $-2 a$, which is below $b$. Hence, equations

$$
x=\mu_{2}\left(a, u_{2}, u_{3}\right) t, \quad x=\mu_{3}\left(a, u_{2}, u_{3}\right) t
$$

can be inverted to give $u_{2}$ and $u_{3}$ as functions of $x / t$ in the region $\mu_{2}(-a / 4,-a / 4, a)<$ $x / t<\mu_{2}(-2 a,-2 a, a)$. To the right of this region, the Burgers type equation (1.5) has a rarefaction wave solution.

Acknowledgments. We would like to thank Yuji Kodama and David Levermore for fruitful discussions. We are also grateful to the referees for valuable comments. V.P. was supported in part by NSF Grant DMS-0135308 and F.-R. T. was supported in part by NSF Grant DMS-0404931.

\section{REFERENCES}

[1] B. A. Dubrovin and S. P. Novikov, Hydrodynamics of weakly deformed soliton lattices. Differential Geometry and Hamiltonian Theory, Russian Math. Surveys 44, 6, 35-124, 1989

[2] A. V. Gurevich and L. P. Pitaevskii, Non-stationary structure of a collisionless shock wave, Soviet Phys. JETP, 38, 291-297, 1974

[3] I. M. Krichever, The method of averaging for two-dimensional 'integrable' Equations, Functional Anal. App., 22, 200-213, 1988.

[4] P. D. Lax and C. D. Levermore, The small dispersion limit for the Korteweg-de Vries equation I, II, and III, Comm. Pure Appl. Math., 36, 253-290, 571-593, 809-830, 1983.

[5] P. D. Lax, C. D. Levermore and S. Venakides, The generation and propagation of oscillations in dispersive IVPs and their limiting behavior, Important Developments in Soliton Theory 1980-1990, T. Fokas and V. E. Zakharov eds., Springer-Verlag, Berlin 1992.

[6] P. G. LeFloch, Hyperbolic Systems of Conservation Laws, Lectures in Mathematics, Birkhauser, 2002.

[7] C. D. Levermore, The hyperbolic nature of the zero dispersion KdV limit, Comm. P. D. E. 13, 495-514, 1988.

[8] F.-R. Tian, Oscillations of the zero dispersion limit of the Korteweg-de Vries equation, Comm. Pure Appl. Math., 46, 1093-1129, 1993.

[9] F.-R. Tian, On the initial value problem of the Whitham averaged system, Singular Limits of Dispersive Waves, N. Ercolani, I. Gabitov, D. Levermore and D. Serre eds., NATO ARW series, Series B: Physics, Plenum, New York, 320, 135-141, 1994.

[10] F.-R. Tian, The Whitham type equations and linear overdetermined systems of Euler-PoissonDarboux type, Duke Math. J., 74, 203-221, 1994.

[11] S. P. Tsarev, Poisson brackets and one-dimensional Hamiltonian systems of hydrodynamic type, Soviet Math. Dokl., 31, 488-491, 1985.

[12] S. Venakides, The zero dispersion limit of the KdV equation with nontrivial reflection coefficient, Comm. Pure Appl. Math. 38, 125-155, 1985.

[13] G. B. Whitham, Non-linear Dispersive Waves, Proc. Royal Soc. London Ser. A, 139, 283-291, 1965. 